\title{
A Systematic Investigation of the Synthetic Utility of Glycopeptide Glycosyltransferases
}

\author{
Markus Oberthür, Catherine Leimkuhler, Ryan G. Kruger, Wei Lu, Christopher T. Walsh and Daniel \\ Kahne* \\ Department of Chemistry and Chemical Biology, Harvard University, Cambridge, MA 02138 \\ Department of Biological Chemistry and Molecular Pharmacology, Harvard Medical School, \\ Boston, MA 02115 \\ kahne@chemistry.harvard.edu
}

\section{Supporting Information}

\section{References:}

(3) (a) Chang, S.; Sievert, D. M.; Hageman, J. C.; Boulton, M. L.; Tenover, F. C.; Downes, F. P.; Shah, S.; Rudrik, J. T.; Pupp, G. R.; Brown, W. J.; Cardo, D.; Fridkin, S. K. N. Engl. J. Med. 2003, $348,1342-1347$.

(13) (e) Ostash, B.; Rix, U.; Rix, L. L.; Liu, T.; Lombo, F.; Luzhetskyy, A.; Gromyko, O.; Wang, C.; Braña, A. F.; Méndez, C.; Salas, J. A.; Fedorenko, V.; Rohr, J. Chem. Biol. 2004, 11, 547-555.

General Methods. All chemicals were purchased from Aldrich or Sigma and used without further purification. Solvents were reagent grade and were further dried when necessary. Analytical thin-layer chromatography was performed on glass plates precoated with silica gel $(250 \mu \mathrm{m}$, Sorbent Technologies), with detection by UV and/or spraying with $\mathrm{H}_{2} \mathrm{SO}_{4}(50 \%)$. Flash chromatography was 


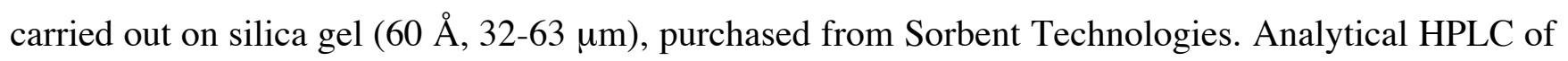
reaction mixtures was performed on a Hewlett-Packard 1100 series instrument using a Phenomenex Luna $5 \mu \mathrm{m} \mathrm{C18} \mathrm{column} \mathrm{(250} \mathrm{mm} \times 4.6 \mathrm{~mm})$. Compounds bearing a thymidine chromophore were monitored at an absorbance of $270 \mathrm{~nm}$. Reactions were monitored by HPLC using method A (linear gradient from $\mathrm{H}_{2} \mathrm{O} / 0.1 \% \mathrm{NH}_{4} \mathrm{HCO}_{3}$ to $100 \% \mathrm{MeOH} / 0.1 \% \mathrm{NH}_{4} \mathrm{HCO}_{3}$ over the course of 20 min, flowrate: $1 \mathrm{~mL} / \mathrm{min}$ ). Preparative HPLC was performed on a Varian ProStar instrument (flow-rate: 45 $\mathrm{mL} / \mathrm{min}$ ) using a Phenomenex Luna $10 \mu \mathrm{m}$ C18 column (250 x $50 \mathrm{~mm}$ ) or on a Hitachi L6200 instrument (flow-rate: $7.5 \mathrm{~mL} / \mathrm{min})$ using a Phenomenex Luna $5 \mu \mathrm{m}$ C18 column $(250 \times 21.2 \mathrm{~mm})$. NMR spectra were recorded on Varian Mercury $300 \mathrm{MHz}$ and Inova 400 or $500 \mathrm{MHz}$ spectrometers. Peaks in the ${ }^{1} \mathrm{H}$ NMR spectra were assigned using COSY experiments. Mass spectra (ESI) were obtained at the Mass Spectroscopy Facility at the Department of Chemistry, Princeton University.

\section{Synthesis of TDP sugars $3-12$ :}

TDP $\beta$-L-vancosamine (3), TDP 4-epi- $\beta$-L-vancosamine (4), TDP $\beta$-L-daunosamine (5), TDP $\beta$-Lacosamine (6), and TDP 2-deoxy- $\beta$-L-fucose (9) were synthesized as previously described. ${ }^{\mathrm{S} 1}$ TDP 4-amino-2,4-deoxy- $\beta$-L-fucose (7):

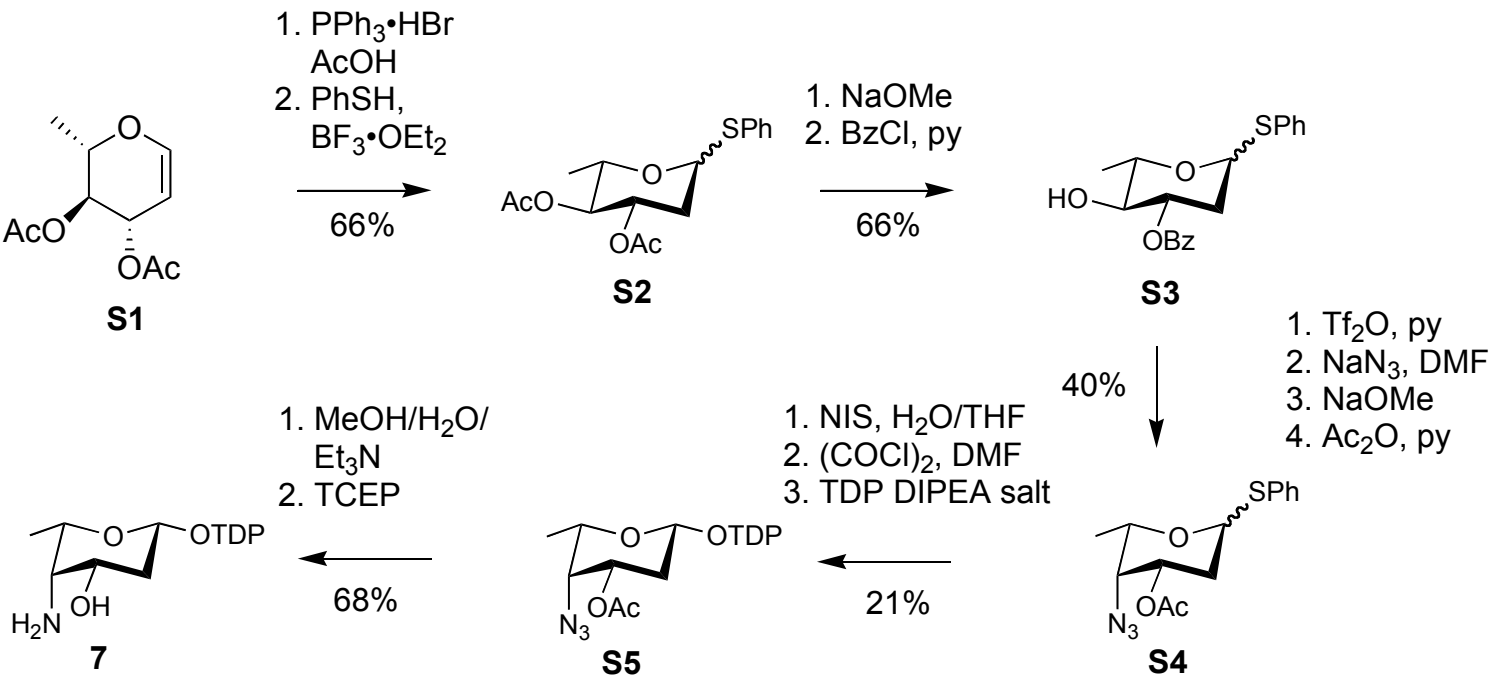


Phenyl 3,4-di-O-acetyl-2-deoxy-1-thio-L-rhamnopyranoside (Phenyl 3,4-di-O-acetyl-2,6-deoxy-1-thioL-arabino-hexopyranoside, S2): To a solution of 3,4-Di- $O$-acetyl-L-rhamnal $(2.51 \mathrm{~g}, 11.7 \mathrm{mmol}, \mathbf{S 1}$, Aldrich) in $\mathrm{CH}_{2} \mathrm{Cl}_{2}(60 \mathrm{~mL})$ was added $\mathrm{AcOH}(1.1 \mathrm{~mL})$ and $\mathrm{PPh}_{3} \cdot \mathrm{HBr}(0.23 \mathrm{~g}, 0.67 \mathrm{mmol}) .{ }^{\mathrm{S} 2}$ After stirring for $16 \mathrm{~h}$ at room temperature, more $\mathrm{PPh}_{3} \bullet \mathrm{HBr}(0.23 \mathrm{~g}, 0.67 \mathrm{mmol})$ was added, and stirring was continued for $24 \mathrm{~h}$. The solution was evaporated and purified by flash chromatography (petroleum ether/EtOAc 4:1) to afford 1,3,4-tri- $O$-acetyl-L-rhamnopyranose $(2.2 \mathrm{~g}, 72 \%)$ as a colorless syrup (mixture of anomers, $R_{\mathrm{f}}$ (petroleum ether/EtOAc 4:1) $=0.25$. The triacetate was dissolved in $\mathrm{CH}_{2} \mathrm{Cl}_{2}$ $(50 \mathrm{~mL}), \mathrm{PhSH}(1 \mathrm{~mL}, 10 \mathrm{mmol})$ and $\mathrm{BF}_{3} \bullet \mathrm{OEt}_{2}(1.3 \mathrm{~mL}, 10.0 \mathrm{mmol})$ was added, and the mixture was stirred at room temperature for $1 \mathrm{~h}$. The solution was diluted with $\mathrm{CH}_{2} \mathrm{Cl}_{2}(250 \mathrm{~mL})$, washed with satd. aq. $\mathrm{NaHCO}_{3}$ solution, aq. $\mathrm{NaOH}$ solution $(10 \%)$, and brine. The organic phase was dried $\left(\mathrm{MgSO}_{4}\right)$, filtered, evaporated, and purified by flash chromatography (petroleum ether/EtOAc 6:1 $\rightarrow$ 4:1) to give thioglycoside S2 (2.5 g, 92\%) as a colorless syrup (mixture of anomers, $R_{\mathrm{f}}$ (petroleum ether/EtOAc 4:1) $=0.52)$; LRMS (ESI) for $\mathrm{C}_{16} \mathrm{H}_{20} \mathrm{O}_{5} \mathrm{~S}(324.39): 325[\mathrm{M}+\mathrm{H}]^{+}$.

${ }^{1} \mathrm{H}$ NMR (400 MHz, $\left.\mathrm{CDCl}_{3}\right): \delta$ 7.45-7.20 (m, 5H, Ph), $5.56\left(\mathrm{~d}, J_{1,2 \mathrm{~b}}=5.8 \mathrm{~Hz}, 1 \alpha-\mathrm{H}\right), 5.23\left(\mathrm{ddd}, J_{2 \mathrm{a}, 3}=\right.$ $\left.5.1, J_{2 \mathrm{~b}, 3}=11.7, J_{3,4}=9.3 \mathrm{~Hz}, 3 \alpha-\mathrm{H}\right), 4.94\left(\mathrm{ddd}, J_{2 \mathrm{a}, 3}=11.4, J_{2 \mathrm{~b}, 3}=5.4, J_{3,4}=9.4 \mathrm{~Hz}, 3 \beta-\mathrm{H}\right), 4.78-4.68$ $(\mathrm{m}, 1 \beta-\mathrm{H}, 4 \alpha-\mathrm{H}, 4 \beta-\mathrm{H}), 4.35\left(\mathrm{dq}, J_{4,5}=9.7, J_{5,6}=6.1 \mathrm{~Hz}, 5 \alpha-\mathrm{H}\right), 3.45\left(\mathrm{dq}, J_{4,5}=9.6, J_{5,6}=6.2 \mathrm{~Hz}, 5 \beta-\right.$ $\mathrm{H}), 2.22-2.18\left(\mathrm{~m}, 2 \alpha-\mathrm{H}_{\mathrm{a}}, 2 \beta-\mathrm{H}_{\mathrm{a}}\right), 2.15\left(\mathrm{ddd}, J_{1,2 \mathrm{~b}}=5.8, J_{2 \mathrm{a}, 2 \mathrm{~b}}=13.0, J_{2 \mathrm{~b}, 3}=11.7 \mathrm{~Hz}, 2 \alpha-\mathrm{H}_{\mathrm{b}}\right), 2.04,2.01$, $1.99,1.97(4 \mathrm{~s}, \mathrm{Ac}), 1.80\left(\right.$ app. q, $\left.J_{1,2 \mathrm{~b}}=11.9, J_{2 \mathrm{a}, 2 \mathrm{~b}}=13.0, J_{2 \mathrm{~b}, 3}=11.4 \mathrm{~Hz}, 2 \beta-\mathrm{H}_{\mathrm{b}}\right), 1.22\left(\mathrm{~d}, J_{5,6}=6.2 \mathrm{~Hz}\right.$, $\left.6 \beta-\mathrm{H}_{3}\right), 1.15\left(\mathrm{~d}, J_{5,6}=6.1 \mathrm{~Hz}, 6 \alpha-\mathrm{H}_{3}\right)$.

Phenyl 3-O-benzoyl-2-deoxy-1-thio-L-rhamnopyranoside (Phenyl 3-O-benzoyl-2,6-deoxy-1-thio-Larabino-hexopyranoside, $\mathbf{S 3})$ : Thioglycoside $\mathbf{S 2}(650 \mathrm{mg}, 2.0 \mathrm{mmol})$ was dissolved in $\mathrm{MeOH}(30 \mathrm{~mL})$ and treated with $\mathrm{NaOMe}(0.3 \mathrm{mmol}, 0.6 \mathrm{~mL}, 0.5 \mathrm{M}$ in $\mathrm{MeOH})$. After stirring for $2 \mathrm{~h}$ at room 
temperature, the solution was neutralized with Amberlite IR120 $\left(\mathrm{H}^{+}\right.$form $)$, filtered, evaporated, and coevaporated with toluene $(3 \mathrm{x})$ to afford the deacetylated product ( $280 \mathrm{mg}$, quant.) as a colorless syrup (mixture of anomers, $R_{\mathrm{f}}$ (petroleum ether/EtOAc 2:1) $\left.=0.10\right)$. The diol was dissolved in pyridine $(10$ $\mathrm{mL})$ and cooled to $-10^{\circ} \mathrm{C}$. Benzoyl chloride $(0.23 \mathrm{~mL}, 2.0 \mathrm{mmol})$ was added dropwise over $3 \mathrm{~h}$ at this temperature. After $10 \mathrm{~min}, \mathrm{MeOH}(10 \mathrm{~mL})$ was added, and the mixture was evaporated. The residue was dissolved in with $\mathrm{CH}_{2} \mathrm{Cl}_{2}(100 \mathrm{~mL})$, washed with $1 \mathrm{~N} \mathrm{HCl}$, satd. aq. $\mathrm{NaHCO}_{3}$ solution, and brine. The organic phase was dried $\left(\mathrm{MgSO}_{4}\right)$, filtered, evaporated, and purified by flash chromatography (petroleum ether/EtOAc 6:1) to give the 3-benzoate $\mathbf{S 3}(455 \mathrm{mg}, 66 \%$ ) as a colorless syrup (mixture of anomers, $R_{\mathrm{f}}$ (petroleum ether/EtOAc 4:1) = 0.45 and 0.50); LRMS (ESI) for $\mathrm{C}_{19} \mathrm{H}_{20} \mathrm{O}_{4} \mathrm{~S}(344.42): 345$ $[\mathrm{M}+\mathrm{H}]^{+}$.

S3a: ${ }^{1} \mathrm{H}$ NMR (400 MHz, $\left.\mathrm{CDCl}_{3}\right): \delta 8.10-7.20(\mathrm{~m}, 10 \mathrm{H}, \mathrm{Ph}), 5.62\left(\mathrm{dd}, 1 \mathrm{H}, J_{1,2 \mathrm{a}}=1.2, J_{1,2 \mathrm{~b}}=5.2 \mathrm{~Hz}, 1-\right.$ H), $5.34\left(\mathrm{ddd}, 1 \mathrm{H}, J_{2 \mathrm{a}, 3}=5.1, J_{2 \mathrm{~b}, 3}=11.7, J_{3,4}=9.1 \mathrm{~Hz}, 3-\mathrm{H}\right), 4.29\left(\mathrm{dq}, 1 \mathrm{H}, J_{4,5}=9.3, J_{5,6}=6.2 \mathrm{~Hz}, 5-\mathrm{H}\right)$, $3.45\left(\mathrm{t}, 1 \mathrm{H}, J_{3,4}=J_{4,5}=9.3 \mathrm{~Hz}, 4-\mathrm{H}\right), 2.80(\mathrm{bs}, 1 \mathrm{H}, 4-\mathrm{OH}), 2.52\left(\mathrm{ddd}, J_{1,2}=1.2, J_{2 \mathrm{a}, 2 \mathrm{~b}}=13.2, J_{2 \mathrm{a}, 3}=5.2\right.$ $\left.\mathrm{Hz}, 2-\mathrm{H}_{\mathrm{a}}\right), 2.28\left(\mathrm{ddd}, J_{1,2 \mathrm{~b}}=5.8, J_{2 \mathrm{a}, 2 \mathrm{~b}}=13.2, J_{2 \mathrm{~b}, 3}=11.7 \mathrm{~Hz}, 2-\mathrm{H}_{\mathrm{b}}\right), 1.35\left(\mathrm{~d}, J_{5,6}=6.2 \mathrm{~Hz}, 6-\mathrm{H}_{3}\right)$.

Phenyl 3-O-acetyl-4-azido-2,4-deoxy-1-thio-L-fuccopyranoside (Phenyl 3-O-acetyl-4-azido-2,4,6deoxy-1-thio-L-lyxo-hexopyranoside, $\mathbf{S 4}$ ): To a solution of alcohol $\mathbf{S 3}$ (453 mg, $1.32 \mathrm{mmol}$ ) in $\mathrm{CH}_{2} \mathrm{Cl}_{2}$ $(10 \mathrm{~mL})$ was added pyridine $(0.9 \mathrm{~mL})$ and triflic anhydride $\left(\mathrm{Tf}_{2} \mathrm{O}\right)(0.44 \mathrm{~mL}, 2.6 \mathrm{mmol})$ at $0^{\circ} \mathrm{C}$. After stirring at room temperature for $1 \mathrm{~h}$, the reaction was diluted with $\mathrm{CH}_{2} \mathrm{Cl}_{2}(100 \mathrm{~mL})$ and washed successively with $1 \mathrm{~N} \mathrm{HCl}$ and brine, dried $\left(\mathrm{MgSO}_{4}\right)$, filtered, and evaporated. The dark residue was dissolved in DMF (5 mL), $\mathrm{NaN}_{3}(600 \mathrm{mg}, 9.21 \mathrm{mmol})$ was added, and the mixture was stirred at room temperature for $16 \mathrm{~h}$. The residue obtained after evaporation was partitioned between $\mathrm{H}_{2} \mathrm{O}$ and EtOAc (100 mL each), and the aq. phase was extracted with EtOAc $(3 \times 50 \mathrm{~mL})$. The combined org. phases were dried $\left(\mathrm{MgSO}_{4}\right)$, filtered, evaporated, and purified by flash chromatography (petroleum ether/EtOAc 15:1, then 9:1) to afford the 4-azido derivative (250 mg, 52\%) as a mixture of anomers, $R_{\mathrm{f}}$ 
(petroleum ether/EtOAc 4:1) =0.25 ( $\beta$-anomer) and 0.60 ( $\alpha$-anomer). This compound $(250 \mathrm{mg}, 0.68$ mmol) was dissolved in $\mathrm{MeOH}(10 \mathrm{~mL}), \mathrm{NaOMe}(0.6 \mathrm{mmol}, 1.2 \mathrm{~mL}, 0.5 \mathrm{M}$ in $\mathrm{MeOH})$ was added, and the solution was refluxed for $2 \mathrm{~h}$. After cooling to room temperature, the solution was neutralized with Amberlite IR120 ( $\mathrm{H}^{+}$form $)$, filtered, evaporated, and coevaporated with toluene $(3 \mathrm{x})$. The residue was dissolved in $\mathrm{CH}_{2} \mathrm{Cl}_{2}(10 \mathrm{~mL})$ and $\mathrm{Et}_{3} \mathrm{~N}(0.39 \mathrm{~mL} 2.8 \mathrm{mmol})$, acetic anhydride $(0.14 \mathrm{~mL}, 1.4 \mathrm{mmol})$ and DMAP (20 mg) was added. After stirring for $5 \mathrm{~h}$ at room temperature, the solution was diluted with $\mathrm{CH}_{2} \mathrm{Cl}_{2}(100 \mathrm{~mL})$, washed with satd. aq. $\mathrm{NaHCO}_{3}$ solution and brine, dried $\left(\mathrm{MgSO}_{4}\right)$, filtered, and evaporated. Purification of the residue by flash chromatography (petroleum ether/EtOAc 6:1) gave thioglycoside S4 (158 mg, 76\%) as a colorless syrup (mixture of anomers, $R_{\mathrm{f}}$ (petroleum ether/EtOAc $4: 1)=0.57$ and 0.60); LRMS (ESI) for $\mathrm{C}_{14} \mathrm{H}_{17} \mathrm{~N}_{3} \mathrm{O}_{3} \mathrm{~S}(307.37): 308[\mathrm{M}+\mathrm{H}]^{+}$.

S4a: ${ }^{1} \mathrm{H}$ NMR $\left(300 \mathrm{MHz}, \mathrm{CDCl}_{3}\right): \delta$ 7.43-7.18 (m, 5H, Ph), $5.67\left(\mathrm{~d}, 1 \mathrm{H}, J_{1,2 \mathrm{a}}=5.8 \mathrm{~Hz}, 1-\mathrm{H}\right), 5.31$ (ddd, $\left.1 \mathrm{H}, J_{2 \mathrm{a}, 3}=12.8, J_{2 \mathrm{~b}, 3}=4.5, J_{3,4}=3.4 \mathrm{~Hz}, 3-\mathrm{H}\right), 4.49\left(\mathrm{dq}, 1 \mathrm{H}, J_{4,5}=1.0, J_{5,6}=6.4 \mathrm{~Hz}, 5-\mathrm{H}\right), 3.83(\mathrm{~m}, 1 \mathrm{H}$, 4-H), $2.50\left(\mathrm{dt}, 1 \mathrm{H}, J_{1,2}=5.8, J_{2 \mathrm{a}, 2 \mathrm{~b}}=J_{2 \mathrm{a}, 3}=12.8 \mathrm{~Hz}, 2-\mathrm{H}_{\mathrm{a}}\right), 2.08-2.00\left(\mathrm{~m}, 4 \mathrm{H}, 2-\mathrm{H}_{\mathrm{b}}, \mathrm{Ac}\right), 1.26\left(\mathrm{~d}, J_{5,6}=\right.$ $\left.6.4 \mathrm{~Hz}, 6-\mathrm{H}_{3}\right)$.

TDP 3-O-acetyl-4-azido-2,4-deoxy- $\beta$-L-fucose (Thymidine 5'-(3-O-acetyl-4-azido-2,4,6-deoxy- $\beta$ - $L$ lyxo-hexopyranosyl diphosphate), S5): Thioglycoside $\mathbf{S 4}(79 \mathrm{mg}, 260 \mu \mathrm{mol})$ was dissolved in $\mathrm{CH}_{3} \mathrm{CN}$ (4 mL) and $\mathrm{H}_{2} \mathrm{O}(0.4 \mathrm{~mL})$ and $N$-iodosuccinimide (NIS) $(75 \mathrm{mg}, 330 \mu \mathrm{mol})$ was added. The solution was stirred for 30 min, quenched with $10 \%$ aq. $\mathrm{Na}_{2} \mathrm{~S}_{2} \mathrm{O}_{3}$ solution, diluted with EtOAc $(100 \mathrm{~mL})$ and washed with brine. The organic phase was dried $\left(\mathrm{MgSO}_{4}\right)$, filtered, evaporated, and purified by flash chromatography (petroleum ether/EtOAc $4: 1$, then $1: 1)$ to afford the lactol (49 $\mathrm{mg}, 87 \%)$ as a colorless syrup (mixture of anomers, $R_{\mathrm{f}}$ (petroleum ether/EtOAc $\left.2: 1\right)=0.60$ ).

The lactol $(19 \mathrm{mg}, 90 \mu \mathrm{mol})$ was dissolved in $\mathrm{CH}_{2} \mathrm{Cl}_{2}(3 \mathrm{~mL})$, freshly activated molecular sieve $(3 \AA)$ was added, and the mixture was stirred for 30 min under argon. A catalytic amount of DMF (5 $\mu \mathrm{L})$ and oxalyl chloride (54 $\mu \mathrm{L}, 110 \mu \mathrm{mol}, 2 \mathrm{M}$ in $\mathrm{CH}_{2} \mathrm{Cl}_{2}$ ) was added, and stirring was continued for $1 \mathrm{~h}$. The 
reaction mixture was filtered, evaporated, and coevaporated with toluene $(3 \mathrm{x})$ without heating. The residue was dissolved in $\mathrm{CH}_{3} \mathrm{CN}(1 \mathrm{~mL})$ under argon, and the $\mathrm{pH}$ was raised to $>7$ by the dropwise addition of DIPEA. This solution was added at $0^{\circ} \mathrm{C}$ to a solution of TDP DIPEA salt in $\mathrm{CH}_{3} \mathrm{CN}$ (1.5 mL) containing freshly activated molecular sieve (3Å). (TDP DIPEA salt: the sodium salt of TDP (50 mg, $120 \mu \mathrm{mol}$, Sigma) was dissolved in $\mathrm{CH}_{3} \mathrm{CN} / \mathrm{H}_{2} \mathrm{O}$ (5:1, $3 \mathrm{~mL}$ ), DOWEX 50WX8-200 (DIPEA form) was added at $0^{\circ} \mathrm{C}$ and the mixture was stirred at this temperature for $1 \mathrm{~h}$. The mixture was filtered, the resin was washed with $\mathrm{CH}_{3} \mathrm{CN}$, and the combined filtrates were evaporated and coevaporated with toluene $(3 \mathrm{x})$ without heating.)

The reaction mixture was stirred for $16 \mathrm{~h}$ at room temperature, filtered, evaporated, and the resulting mixture of anomers $(\mathbf{S 5} \boldsymbol{\beta} / \mathbf{S 5} \boldsymbol{\alpha}=3: 2)$, was purified by preparative HPLC. Evaporation of the fractions containing the $\beta$-isomer gave $\mathbf{S 5} \beta(13 \mathrm{mg}, 24 \%)$ as a powder upon lyophilization; $R_{\mathrm{t}}(\mathrm{TDP})=3.6 \mathrm{~min}$, $R_{\mathrm{t}}(\mathbf{S 5}, \beta$-anomer $)=9.6 \min , R_{\mathrm{t}}(\mathbf{S 5}, \alpha$-anomer $)=10.0 \mathrm{~min}$, method A; LRMS $(\mathrm{ESI})$ for $\mathrm{C}_{18} \mathrm{H}_{27} \mathrm{~N}_{5} \mathrm{O}_{14} \mathrm{P}_{2}$ (599.38): $598[\mathrm{M}-\mathrm{H}]^{-}$.

${ }^{1} \mathrm{H}$ NMR (400 MHz, CD $\left.\mathrm{OD}\right): \delta 7.86(\mathrm{~s}, 1 \mathrm{H}, 6-\mathrm{H}), 6.23\left(\mathrm{t}, 1 \mathrm{H}, J_{1,2 \mathrm{a}}=J_{1,2 \mathrm{~b}}=6.4 \mathrm{~Hz}, 1{ }^{\prime}-\mathrm{H}\right) 5.27(\mathrm{t}, 1 \mathrm{H}, J-$ $\left.{ }_{1,2}=J_{1, \mathrm{P}}=8.8 \mathrm{~Hz}, 1^{\prime \prime}-\mathrm{H}\right), 5.12\left(\mathrm{ddd}, 1 \mathrm{H}, J_{2,3}=4.8, J_{2,3}=12.4, J_{3,4}=3.5 \mathrm{~Hz}, 3 "-\mathrm{H}\right), 4.61-4.57\left(\mathrm{~m}, 1 \mathrm{H}, 3^{\prime}-\right.$ H), 4.24-4.14 (m, 2H, 5'-H $), 4.03$ (bs, 1H, 4'-H), 3.76-3.72 (m, 1H, 5"-H), 3.32 (bs, $1 \mathrm{H}, 4 "-\mathrm{H})$, 2.36-2.18 (m, 3H, 2'- $\left.\mathrm{H}_{2}, 2^{\prime \prime}-\mathrm{H}_{\mathrm{a}}\right), 2.06(\mathrm{~s}, 3 \mathrm{H}, \mathrm{Ac}), 1.91$ (s, 3H, thymidine $\left.\mathrm{CH}_{3}\right), 1.90-1.80\left(\mathrm{~m}, 1 \mathrm{H}, 2^{\prime \prime}-\right.$ $\left.\mathrm{H}_{\mathrm{b}}\right), 1.28\left(\mathrm{~d}, 3 \mathrm{H}, J_{5,6}=6.4 \mathrm{~Hz}, 6 "-\mathrm{H}_{3}\right) ;{ }^{31} \mathrm{P} \mathrm{NMR}\left(162 \mathrm{MHz}, \mathrm{CD}_{3} \mathrm{OD}\right): \delta-10.3,-12.5$.

TDP 4-amino-2,4-deoxy- $\beta$-L-fucose (Thymidine 5'-(4-amino-2,4,6-deoxy- $\beta$-L-lyxo-hexopyranosyl diphosphate), 7): The protected TDP sugar S5 (5 mg, $8 \mu \mathrm{mol})$ was dissolved in $\mathrm{MeOH} / \mathrm{H}_{2} \mathrm{O} / \mathrm{Et}_{3} \mathrm{~N}(0.5$ $\mathrm{mL}, 2: 2: 1)$ and stirred for $16 \mathrm{~h}$ at room temperature. Tris-(2-carboxyethyl)-phosphine hydrochloride (TCEP hydrochloride) $(15 \mathrm{mg}, 52 \mu \mathrm{mol})$ was added, and stirring was continued for $3 \mathrm{~h}$. The reaction mixture was evaporated and purified by preparative HPLC to afford TDP sugar 7 (3 mg, 68\%) as a 
white powder upon lyophilization; $R_{\mathrm{t}}=6.2 \mathrm{~min}$, method A; LRMS (ESI) for $\mathrm{C}_{16} \mathrm{H}_{27} \mathrm{~N}_{3} \mathrm{O}_{13} \mathrm{P}_{2}$ (531.35): $530[\mathrm{M}-\mathrm{H}]^{-}$.

${ }^{1} \mathrm{H}$ NMR $\left(500 \mathrm{MHz}, \mathrm{D}_{2} \mathrm{O}\right): \delta=7.74(\mathrm{~s}, 1 \mathrm{H}, 6-\mathrm{H}), 6.35\left(\mathrm{t}, 1 \mathrm{H}, J_{1,2 \mathrm{a}}=J_{1,2 \mathrm{~b}}=6.8 \mathrm{~Hz}, 1^{\prime}-\mathrm{H}\right) 5.23(\mathrm{t}, 1 \mathrm{H}, J-$ $\left.{ }_{1,2}=J_{1, \mathrm{P}}=8.8 \mathrm{~Hz}, 1^{\prime \prime}-\mathrm{H}\right), 4.63-4.59\left(\mathrm{~m}, 1 \mathrm{H}, 3^{\prime}-\mathrm{H}\right), 4.24-4.14$ (m, 4H, 4'-H, 5'-- ${ }_{2}, 3$ '-H), 3.93 (q, 1H, $\left.J_{5,6}=6.4 \mathrm{~Hz}, 5 "-\mathrm{H}\right), 3.36(\mathrm{bs}, 1 \mathrm{H}, 4 "-\mathrm{H}), 2.42-2.23\left(\mathrm{~m}, 3 \mathrm{H}, 2^{\prime}-\mathrm{H}_{2}, 2 "-\mathrm{H}_{\mathrm{a}}\right), 1.91$ (s, 3H, thymidine $\mathrm{CH}_{3}$ ), $1.60-1.53\left(\mathrm{~m}, 1 \mathrm{H}, 2 "-\mathrm{H}_{\mathrm{b}}\right), 1.27\left(\mathrm{~d}, 3 \mathrm{H}, J_{5,6}=6.4 \mathrm{~Hz}, 6 "-\mathrm{H}_{3}\right) ;{ }^{31} \mathrm{P}$ NMR $\left(162 \mathrm{MHz}, \mathrm{D}_{2} \mathrm{O}\right): \delta-11.0$, -13.3 . 


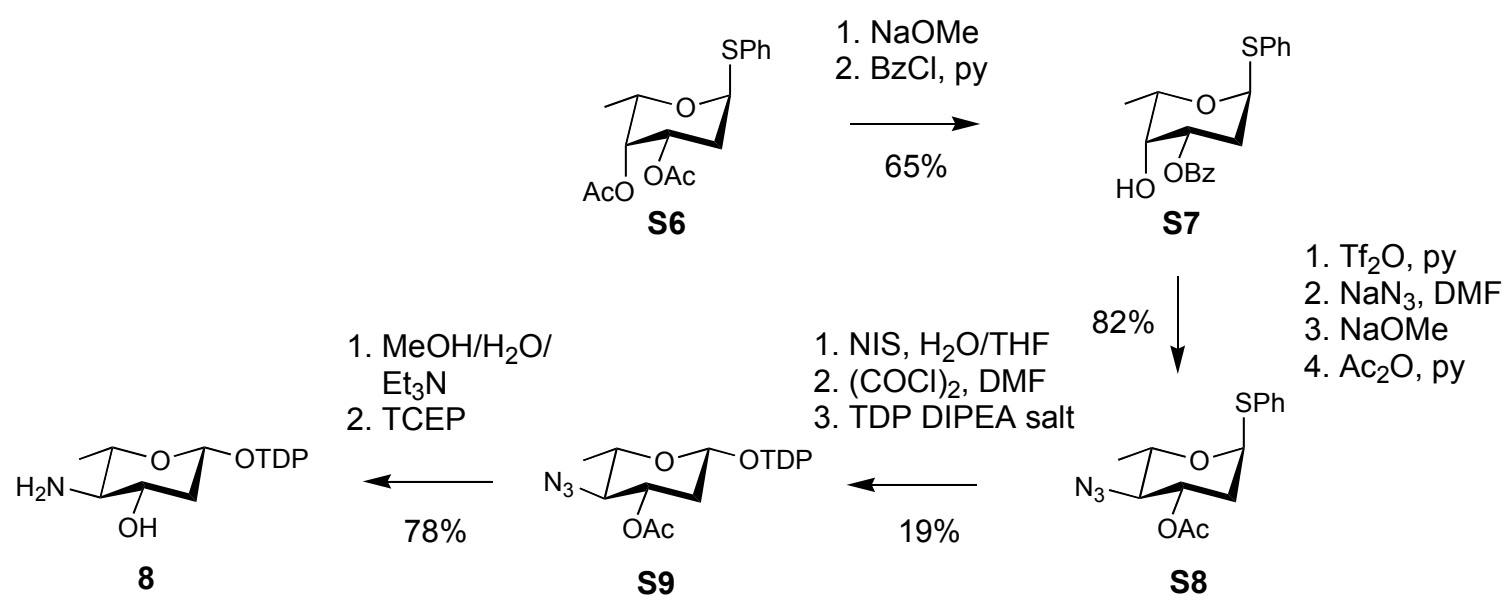

TDP sugar $\mathbf{8}$ was prepared starting from the known thioglycoside $\mathbf{S 6}{ }^{\mathrm{S} 3}$ as described for $\mathbf{7}$.

Phenyl 3-O-benzoyl-2-deoxy-1-thio- $\alpha$-L-fucopyranoside (Phenyl 3-O-benzoyl-2,6-deoxy-1-thio- $\alpha$-Llyxo-hexopyranoside, $\mathbf{S 7}$ ): Thioglycoside $\mathbf{S 6}{ }^{\mathbf{S 3}}$ was converted to the monobenzoate $\mathbf{S 7}$ as described for S3 (65\%, 2 steps); $R_{\mathrm{f}}$ (petroleum ether/EtOAc 4:1) $=0.25$; LRMS (ESI) for $\mathrm{C}_{19} \mathrm{H}_{20} \mathrm{O}_{4} \mathrm{~S}$ (344.42): 345 $[\mathrm{M}+\mathrm{H}]^{+}$.

S3 $\alpha$ : ${ }^{1} \mathrm{H}$ NMR (500 MHz, $\mathrm{CDCl}_{3}$ ): $\delta$ 8.10-7.26 (m, 10H, Ph), $5.77\left(\mathrm{~d}, 1 \mathrm{H}, J_{1,2 \mathrm{a}}=6.0 \mathrm{~Hz}, 1-\mathrm{H}\right), 5.45$ $\left(\mathrm{ddd}, 1 \mathrm{H}, J_{2 \mathrm{a}, 3}=12.8, J_{2 \mathrm{~b}, 3}=5.2, J_{3,4}=2.9 \mathrm{~Hz}, 3-\mathrm{H}\right), 4.58\left(\mathrm{q}, 1 \mathrm{H}, J_{5,6}=6.2 \mathrm{~Hz}, 5-\mathrm{H}\right), 4.01\left(\mathrm{~d}, 1 \mathrm{H}, J_{3,4}=\right.$ $2.9 \mathrm{~Hz}, 4-\mathrm{H}), 2.65\left(\mathrm{ddd}, J_{1,2}=6.0, J_{2 \mathrm{a}, 2 \mathrm{~b}}=J_{2 \mathrm{a}, 3}=12.8 \mathrm{~Hz}, 2-\mathrm{H}_{\mathrm{a}}\right), 2.22\left(\mathrm{dd}, J_{2 \mathrm{a}, 2 \mathrm{~b}}=12.8, J_{2 \mathrm{~b}, 3}=5.2 \mathrm{~Hz}, 2-\right.$ $\left.\mathrm{H}_{\mathrm{b}}\right), 2.07(\mathrm{bs}, 1 \mathrm{H}, 4-\mathrm{OH}), 1.34\left(\mathrm{~d}, J_{5,6}=6.2 \mathrm{~Hz}, 6-\mathrm{H}_{3}\right)$.

Phenyl 3-O-acetyl-4-azido-2,4-deoxy-1-thio- $\alpha$-L-rhamnopyranoside (Phenyl 3-O-acetyl-4-azido-2,4,6deoxy-1-thio- $\alpha$-L-arabino-hexopyranoside, $\mathbf{S 8}$ ): 3-Benzoate $\mathbf{S 7}$ was converted to thioglycoside $\mathbf{S 8}$ using the reaction sequence described for $\mathbf{S 4}$ (82\%, 4 steps); $R_{\mathrm{f}}$ (petroleum ether/EtOAc 9:1) $=0.31$; LRMS (ESI) for $\mathrm{C}_{14} \mathrm{H}_{17} \mathrm{~N}_{3} \mathrm{O}_{3} \mathrm{~S}$ (307.37): $308[\mathrm{M}+\mathrm{H}]^{+}$. 
${ }^{1} \mathrm{H}$ NMR (400 MHz, $\left.\mathrm{CDCl}_{3}\right): \delta 7.44-7.21(\mathrm{~m}, 5 \mathrm{H}, \mathrm{Ph}), 5.55\left(\mathrm{~d}, 1 \mathrm{H}, J_{1,2 \mathrm{~b}}=5.4 \mathrm{~Hz}, 1-\mathrm{H}\right), 5.17(\mathrm{ddd}, 1 \mathrm{H}$, $\left.J_{2 \mathrm{a}, 3}=5.1, J_{2 \mathrm{~b}, 3}=11.7, J_{3,4}=9.8 \mathrm{~Hz}, 3-\mathrm{H}\right), 4.14\left(\mathrm{dq}, 1 \mathrm{H}, J_{4,5}=9.8, J_{5,6}=6.2 \mathrm{~Hz}, 5-\mathrm{H}\right), 3.17\left(\mathrm{t}, 1 \mathrm{H}, J_{3,4}=\right.$ $\left.J_{4,5}=9.8 \mathrm{~Hz}, 4-\mathrm{H}\right), 2.45\left(\mathrm{dd}, 1 \mathrm{H}, J_{2 \mathrm{a}, 2 \mathrm{~b}}=13.2, J_{2 \mathrm{a}, 3}=5.1 \mathrm{~Hz}, 2-\mathrm{H}_{\mathrm{a}}\right), 2.08-2.00\left(\mathrm{~m}, 4 \mathrm{H}, 2-\mathrm{H}_{\mathrm{b}}, \mathrm{Ac}\right), 1.31$ $\left(\mathrm{d}, J_{5,6}=6.2 \mathrm{~Hz}, 6-\mathrm{H}_{3}\right)$.

TDP 3-O-acetyl-4-azido-2,4-deoxy- $\beta$-L-rhamnose (Thymidine 5'-(3-O-acetyl-4-azido-2,4,6-deoxy- $\beta$-Larabino-hexopyranosyl diphosphate), S9): Thioglycoside S8 (30 mg, $96 \mu \mathrm{mol})$ was converted to TDP derivative S9 as described for S5. The resulting mixture of anomers $(\mathbf{S 9} \beta / \mathbf{S 9} \boldsymbol{\alpha}=3: 1)$, was purified by preparative HPLC. Evaporation of the fractions containing the $\beta$-isomer gave $\mathbf{S 9 \beta}$ (11 mg, 19\%) as a powder upon lyophilization; $R_{\mathrm{t}}(\mathrm{TDP})=3.6 \mathrm{~min}, R_{\mathrm{t}}(\mathbf{S 9}, \beta$-anomer $)=9.9 \min , R_{\mathrm{t}}(\mathbf{S 9}$, $\alpha$-anomer $)=9.6$ min, method A; LRMS (ESI) for $\mathrm{C}_{18} \mathrm{H}_{27} \mathrm{~N}_{5} \mathrm{O}_{14} \mathrm{P}_{2}$ (599.38): $598[\mathrm{M}-\mathrm{H}]^{-}$.

${ }^{1} \mathrm{H}$ NMR (300 MHz, CD $\left.\mathrm{OD}\right): \delta 7.82(\mathrm{~s}, 1 \mathrm{H}, 6-\mathrm{H}), 6.32\left(\mathrm{t}, 1 \mathrm{H}, J_{1,2 \mathrm{a}}=J_{1,2 \mathrm{~b}}=6.4 \mathrm{~Hz}, 11^{\prime}-\mathrm{H}\right) 5.31(\mathrm{dt}, 1 \mathrm{H}$, $\left.J_{1,2 \mathrm{a}}=2.0, J_{1,2 \mathrm{~b}}=J_{1, \mathrm{P}}=9.2 \mathrm{~Hz}, 1 "-\mathrm{H}\right), 5.00\left(\mathrm{ddd}, 1 \mathrm{H}, J_{2 \mathrm{a}, 3}=5.0, J_{2 \mathrm{~b}, 3}=12.0, J_{3,4}=9.7 \mathrm{~Hz}, 3 "-\mathrm{H}\right), 4.58(\mathrm{dt}$, $\left.1 \mathrm{H}, J_{2,3}=3.1, J_{2,3}=J_{3,4}=6.0 \mathrm{~Hz}, 3^{\prime}-\mathrm{H}\right), 4.26-4.11\left(\mathrm{~m}, 2 \mathrm{H}, 5^{\prime}-\mathrm{H}_{2}\right), 4.03-3.98\left(\mathrm{~m}, 1 \mathrm{H}, 4^{\prime}-\mathrm{H}\right), 3.39$ (dq, $\left.1 \mathrm{H}, J_{4,5}=9.7, J_{5,6}=6.1 \mathrm{~Hz}, 5 "-\mathrm{H}\right), 3.17\left(\mathrm{t}, 1 \mathrm{H}, J_{3,4}=J_{4,5}=9.7 \mathrm{~Hz}, 4 "-\mathrm{H}\right), 2.52\left(\mathrm{ddd}, 1 \mathrm{H}, J_{1,2 \mathrm{a}}=2.0, J_{2,2}\right.$ $\left.=12.0, J_{2 \mathrm{a}, 3}=5.0 \mathrm{~Hz}, 2^{\prime \prime}-\mathrm{H}_{\mathrm{a}}\right), 2.33-2.15\left(\mathrm{~m}, 2 \mathrm{H}, 2^{\prime}-\mathrm{H}_{2}\right), 2.06(\mathrm{~s}, 3 \mathrm{H}, \mathrm{Ac}), 1.94\left(\mathrm{~s}, 3 \mathrm{H}\right.$, thymidine $\left.\mathrm{CH}_{3}\right)$, $1.64\left(\mathrm{dt}, 1 \mathrm{H}, J_{1,2 \mathrm{~b}}=9.2, J_{2,2}=J_{2 \mathrm{~b}, 3}=12.0 \mathrm{~Hz}, 2 "-\mathrm{H}_{\mathrm{b}}\right), 1.32\left(\mathrm{~d}, 3 \mathrm{H}, J_{5,6}=6.1 \mathrm{~Hz}, 6 "-\mathrm{H}_{3}\right) ;{ }^{31} \mathrm{P}$ NMR $(162$ $\left.\mathrm{MHz}, \mathrm{CD}_{3} \mathrm{OD}\right): \delta-10.0,-12.4$.

TDP 4-amino-2,4-deoxy- $\beta$-L-rhamnose (Thymidine 5'-(4-amino-2,4,6-deoxy- $\beta$-L-arabinohexopyranosyl diphosphate), 8): The protected TDP sugar S9 (7 mg, $12 \mu \mathrm{mol})$ was dissolved in $\mathrm{MeOH} / \mathrm{H}_{2} \mathrm{O} / \mathrm{Et}_{3} \mathrm{~N}(0.5 \mathrm{~mL}, 2: 2: 1)$ and stirred for $16 \mathrm{~h}$ at room temperature. TCEP hydrochloride (22 $\mathrm{mg}, 78 \mu \mathrm{mol}$ ) was added, and stirring was continued for $3 \mathrm{~h}$. The reaction mixture was evaporated and 
purified by preparative HPLC to afford TDP sugar $8(5 \mathrm{mg}, 78 \%)$ as a white powder upon lyophilization; $R_{\mathrm{t}}=6.4$ min, method A; LRMS (ESI) for $\mathrm{C}_{16} \mathrm{H}_{27} \mathrm{~N}_{3} \mathrm{O}_{13} \mathrm{P}_{2}$ (531.35): $530[\mathrm{M}-\mathrm{H}]^{-}$.

${ }^{1} \mathrm{H}$ NMR $\left(500 \mathrm{MHz}, \mathrm{D}_{2} \mathrm{O}\right): \delta 7.61(\mathrm{~s}, 1 \mathrm{H}, 6-\mathrm{H}), 6.21\left(\mathrm{t}, 1 \mathrm{H}, J_{1,2 \mathrm{a}}=J_{1,2 \mathrm{~b}}=6.4 \mathrm{~Hz}, 1{ }^{\prime}-\mathrm{H}\right) 5.15(\mathrm{ddd}, 1 \mathrm{H}, J-$ $\left.{ }_{1,2 \mathrm{a}}=2.0, J_{1,2 \mathrm{~b}}=J_{1, \mathrm{P}}=8.9 \mathrm{~Hz}, 1^{\prime \prime}-\mathrm{H}\right), 4.60-4.52\left(\mathrm{~m}, 3^{\prime}-\mathrm{H}\right), 4.20-4.00\left(2 \mathrm{~m}, 3 \mathrm{H}, 4^{\prime}-\mathrm{H}, 5^{\prime}-\mathrm{H}_{2}\right), 3.73(\mathrm{ddd}$, $\left.1 \mathrm{H}, J_{2 \mathrm{a}, 3}=5.1, J_{2 \mathrm{~b}, 3}=12.0, J_{3,4}=9.6 \mathrm{~Hz}, 3^{\prime \prime}-\mathrm{H}\right), 3.59\left(\mathrm{dq}, 1 \mathrm{H}, J_{4,5}=9.6, J_{5,6}=6.2 \mathrm{~Hz}, 5 "-\mathrm{H}\right), 2.65(\mathrm{t}, 1 \mathrm{H}$, $\left.J_{3,4}=J_{4,5}=9.6 \mathrm{~Hz}, 4 "-\mathrm{H}\right), 2.38\left(\mathrm{ddd}, 1 \mathrm{H}, J_{1,2 \mathrm{a}}=2.0, J_{2,2}=12.0, J_{2 \mathrm{a}, 3}=5.1 \mathrm{~Hz}, 2^{\prime \prime}-\mathrm{H}_{\mathrm{a}}\right), 2.25-2.15(\mathrm{~m}$, $\left.2 \mathrm{H}, 2^{\prime}-\mathrm{H}_{2}\right), 1.91\left(\mathrm{~s}, 3 \mathrm{H}\right.$, thymidine $\left.\mathrm{CH}_{3}\right), 1.55\left(\mathrm{dt}, 1 \mathrm{H}, J_{1,2 \mathrm{~b}}=8.9, J_{2,2}=J_{2 \mathrm{~b}, 3}=12.0 \mathrm{~Hz}, 2^{\prime \prime}-\mathrm{H}_{\mathrm{b}}\right), 1.21(\mathrm{~d}$, $\left.3 \mathrm{H}, J_{5,6}=6.2 \mathrm{~Hz}, 6 "-\mathrm{H}_{3}\right) ;{ }^{31} \mathrm{P} \mathrm{NMR}\left(162 \mathrm{MHz}, \mathrm{D}_{2} \mathrm{O}\right): \delta-10.9,-12.9$.

TDP 2-deoxy- $\beta$-L-rhamnose (10):

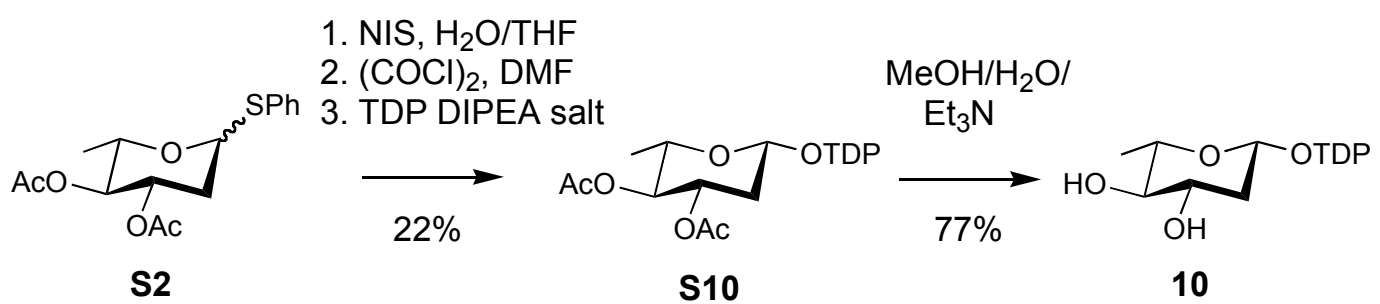

TDP 3,4-di-O-acetyl-2-deoxy- $\beta$-L-rhamnose (Thymidine 5'-(3,4-di-O-acetyl-2,6-deoxy- $\beta$-L-arabinohexopyranosyl diphosphate), S10): TDP sugar S10 was prepared from thioglycoside S2 (21 mg, 66 $\mu \mathrm{mol})$ as described for S5. The resulting mixture of anomers $(\mathbf{S 1 0} \boldsymbol{\beta} / \mathbf{S 1 0} \boldsymbol{\alpha}=2: 1)$, was purified by preparative HPLC. Evaporation of the fractions containing the $\beta$-isomer gave $\mathbf{S 1 0}(9 \mathrm{mg}, 22 \%)$ as a powder upon lyophilization; $R_{\mathrm{t}}(\mathrm{TDP})=3.6 \mathrm{~min}, R_{\mathrm{t}}(\mathbf{S 9}, \beta$-anomer $)=10.0 \mathrm{~min}, R_{\mathrm{t}}(\mathbf{S 9}, \alpha$-anomer $)=9.7$ min, method A; LRMS (ESI) for $\mathrm{C}_{20} \mathrm{H}_{30} \mathrm{~N}_{2} \mathrm{O}_{16} \mathrm{P}_{2}$ (616.40): 615 [M-H] $]^{-}$

${ }^{1} \mathrm{H}$ NMR (400 MHz, CD $\left.3 \mathrm{OD}\right): \delta 7.80(\mathrm{~s}, 1 \mathrm{H}, 6-\mathrm{H}), 6.28\left(\mathrm{t}, 1 \mathrm{H}, J_{1,2 \mathrm{a}}=J_{1,2 \mathrm{~b}}=6.4 \mathrm{~Hz}, 1{ }^{\prime}-\mathrm{H}\right) 5.34(\mathrm{t}, 1 \mathrm{H}, J-$ $\left.{ }_{1,2 \mathrm{~b}}=J_{1, \mathrm{P}}=8.8 \mathrm{~Hz}, 1 "-\mathrm{H}\right), 5.04\left(\mathrm{ddd}, 1 \mathrm{H}, J_{2 \mathrm{a}, 3}=5.2, J_{2 \mathrm{~b}, 3}=12.1, J_{3,4}=9.6 \mathrm{~Hz}, 3^{\prime \prime}-\mathrm{H}\right), 4.60\left(\mathrm{t}, 1 \mathrm{H}, J_{3,4}=\right.$ $\left.J_{4,5}=9.6 \mathrm{~Hz}, 4^{\prime \prime}-\mathrm{H}\right), 4.56-4.50\left(\mathrm{~m}, 1 \mathrm{H}, 3^{\prime}-\mathrm{H}\right), 4.20-4.12\left(\mathrm{~m}, 2 \mathrm{H}, 5^{\prime}-\mathrm{H}_{2}\right), 4.03-3.98$ (m, 1H, 4'-H), 3.52$3.48\left(\mathrm{~m}, 1 \mathrm{H}, 5^{\prime \prime}-\mathrm{H}\right), 2.52-2.46\left(\mathrm{~m}, 1 \mathrm{H}, 2^{\prime \prime}-\mathrm{H}_{\mathrm{a}}\right), 2.28-2.15\left(\mathrm{~m}, 2 \mathrm{H}, 2^{\prime}-\mathrm{H}_{2}\right), 2.00,1.94,1.92(3 \mathrm{~s}$, each $3 \mathrm{H}$, 
2 Ac, thymidine $\left.\mathrm{CH}_{3}\right), 1.64\left(\mathrm{dt}, 1 \mathrm{H}, J_{1,2 \mathrm{~b}}=8.8, J_{2,2}=J_{2 \mathrm{~b}, 3}=12.1 \mathrm{~Hz}, 2^{\prime \prime}-\mathrm{H}_{\mathrm{b}}\right), 1.18\left(\mathrm{~d}, 3 \mathrm{H}, J_{5,6}=6.2 \mathrm{~Hz}\right.$, 6"- $\left.\mathrm{H}_{3}\right) ;{ }^{31} \mathrm{P} \mathrm{NMR}\left(162 \mathrm{MHz}, \mathrm{CD}_{3} \mathrm{OD}\right): \delta-10.4,-12.8$.

TDP 2-deoxy- $\beta$-L-rhamnose (Thymidine 5'-(2,6-deoxy- $\beta$-L-arabino-hexopyranosyl diphosphate), 10): The protected TDP sugar $\mathbf{S 1 0}(9 \mathrm{mg}, 15 \mu \mathrm{mol})$ was dissolved in $\mathrm{MeOH} / \mathrm{H}_{2} \mathrm{O} / \mathrm{Et}_{3} \mathrm{~N}(1 \mathrm{~mL}, 2: 2: 1)$, stirred for $16 \mathrm{~h}$ at room temperature, evaporated, and purified by preparative HPLC to afford $\mathbf{1 0}$ (6 $\mathrm{mg}, 77 \%$ ) as a white powder upon lyophilization; $R_{\mathrm{t}}=6.8 \mathrm{~min}$, method A; LRMS (ESI) for $\mathrm{C}_{16} \mathrm{H}_{26} \mathrm{~N}_{2} \mathrm{O}_{14} \mathrm{P}_{2}$ (532.33): $531[\mathrm{M}-\mathrm{H}]^{-}$.

${ }^{1} \mathrm{H}$ NMR (400 MHz, D $\left.2 \mathrm{O}\right): \delta 7.57(\mathrm{~s}, 1 \mathrm{H}, 6-\mathrm{H}), 6.17\left(\mathrm{t}, 1 \mathrm{H}, J_{1,2 \mathrm{a}}=J_{1,2 \mathrm{~b}}=6.4 \mathrm{~Hz}, 1\right.$ '-H) $5.07\left(\mathrm{dt}, 1 \mathrm{H}, J_{1,2 \mathrm{a}}\right.$ $\left.=2.1, J_{1,2 \mathrm{~b}}=J_{1, \mathrm{P}}=8.4 \mathrm{~Hz}, 1^{\prime \prime}-\mathrm{H}\right), 4.44-4.40\left(\mathrm{~m}, 3^{\prime}-\mathrm{H}\right), 4.08-3.90\left(\mathrm{~m}, 3 \mathrm{H}, 4^{\prime}-\mathrm{H}, 5^{\prime}-\mathrm{H}_{2}\right), 3.52-3.42(\mathrm{~m}, 1 \mathrm{H}$, $\left.3^{\prime \prime-H}\right), 3.26\left(\mathrm{dq}, 1 \mathrm{H}, J_{4,5}=9.3, J_{5,6}=6.2 \mathrm{~Hz}, 5 "-\mathrm{H}\right), 2.86\left(\mathrm{t}, 1 \mathrm{H}, J_{3,4}=J_{4,5}=9.3 \mathrm{~Hz}, 4 "-\mathrm{H}\right), 2.24-2.06$ $\left(\mathrm{m}, 3 \mathrm{H}, 2^{\prime}-\mathrm{H}_{2}, 2^{\prime \prime}-\mathrm{H}_{\mathrm{a}}\right), 1.81\left(\mathrm{~s}, 3 \mathrm{H}\right.$, thymidine $\left.\mathrm{CH}_{3}\right), 1.56-1.48\left(\mathrm{~m}, 1 \mathrm{H}, 2^{\prime \prime}-\mathrm{H}_{\mathrm{b}}\right), 1.11\left(\mathrm{~d}, 3 \mathrm{H}, J_{5,6}=6.2 \mathrm{~Hz}\right.$, $\left.6 "-\mathrm{H}_{3}\right) ;{ }^{31} \mathrm{P}$ NMR $\left(162 \mathrm{MHz}, \mathrm{D}_{2} \mathrm{O}\right): \delta-10.7,-12.8$.

TDP 2-deoxy- $\beta$-L-glucose (11):

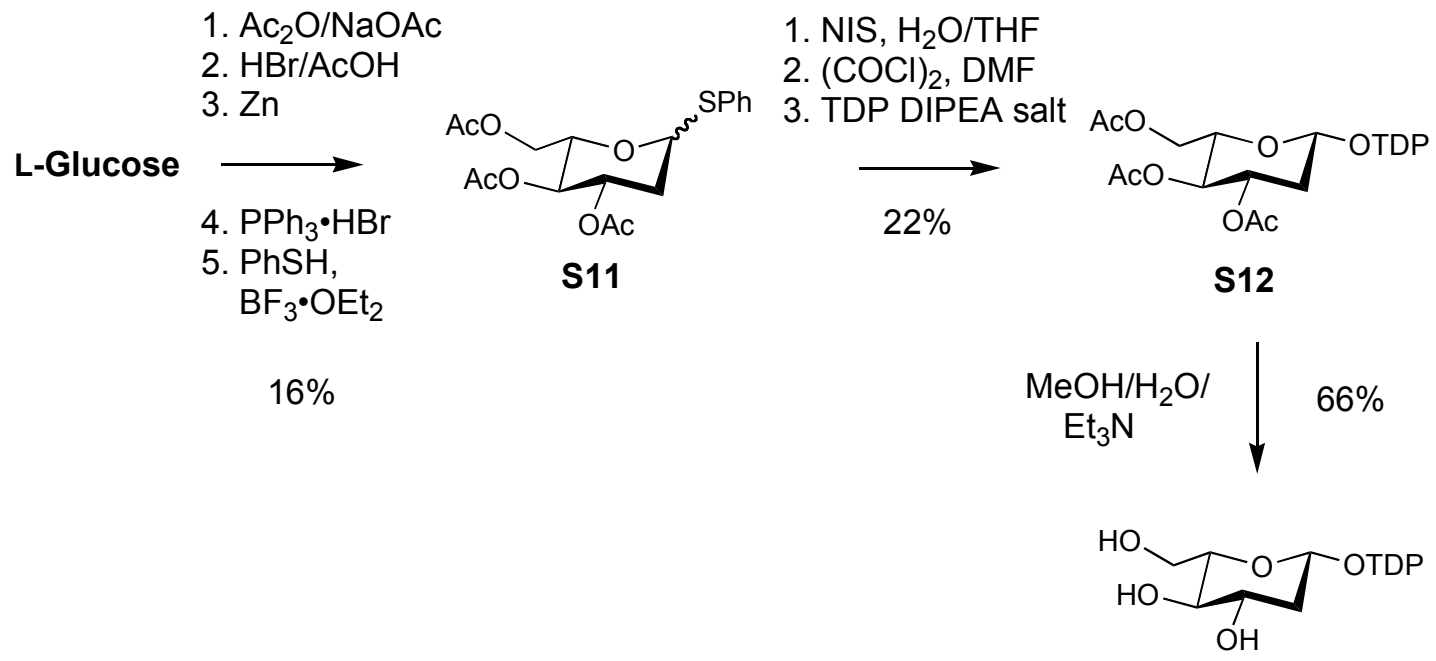

11 
Phenyl 3,4,6-tri-O-acetyl-2-deoxy-1-thio-L-glucopyranoside (S11): A suspension of L-glucose (3.0 g, $17 \mathrm{mmol})$ and $\mathrm{NaOAc}(1.8 \mathrm{~g})$ in acetic anhydride $(30 \mathrm{~mL})$ was stirred at $140^{\circ} \mathrm{C}$ until a clear solution resulted. Stirring was continued for $2 \mathrm{~h}$ at this temperature, and the solution was cooled and poured into ice/water (300 mL). The biphasic mixture was stirred vigorously until precipation of a white solid was complete. The mixture was filtered, and the collected solid was washed with cold water and dried overnight. Recrystallization $\left(\mathrm{EtOH} / \mathrm{H}_{2} \mathrm{O}\right.$ 95:5) afforded the $\beta$-pentaacetate $(3.3 \mathrm{~g}, 50 \%)$ as colorless needles, $R_{\mathrm{f}}$ (petroleum ether/EtOAc 2:1) $=0.50$. The $\beta$-pentaacetate $(2.0 \mathrm{~g}, 5.1 \mathrm{mmol})$ was dissolved in $\mathrm{CH}_{2} \mathrm{Cl}_{2}(20 \mathrm{~mL})$ under argon and $\mathrm{HBr}(5 \mathrm{~mL}, 30 \%$ in $\mathrm{AcOH})$ was added. After $2 \mathrm{~h}$, the reaction mixture was diluted with $\mathrm{CH}_{2} \mathrm{Cl}_{2}(100 \mathrm{~mL})$ and poured into ice/water $(200 \mathrm{~mL})$. The aqueous phase was extracted with $\mathrm{CH}_{2} \mathrm{Cl}_{2}(2 \times 100 \mathrm{~mL})$ and the combined organic phases were washed with $\mathrm{H}_{2} \mathrm{O}$, dried $\left(\mathrm{MgSO}_{4}\right)$, filtered, and evaporated to afford the pure (as judged by TLC) anomeric bromide ( $2.1 \mathrm{~g}$, quant.); $R_{\mathrm{f}}$ (petroleum ether/EtOAc 2:1) $=0.75$. The bromide was dissolved in anhydr. EtOAc $(12 \mathrm{ml})$ and was added to a suspension of zinc powder $(2 \mathrm{~g}, 30 \mathrm{mmol})$ and 1-methylimidazole $(0.4 \mathrm{~mL}, 5$ mmol) in anhydr. EtOAc $(25 \mathrm{~mL})$ at reflux temperature. After 30 min heating to reflux, the mixture was cooled to room temperature, diluted with EtOAc $(200 \mathrm{~mL})$, and filtered through Celite. The filtrate was washed with with $1 \mathrm{~N} \mathrm{HCl}$, satd. aq. $\mathrm{NaHCO}_{3}$ solution, and brine. The organic phase was dried $\left(\mathrm{MgSO}_{4}\right)$, filtered, evaporated, and purified by flash chromatography (petroleum ether/EtOAc 3:1, then 2:1) to give the acetylated L-glucal $(0.9 \mathrm{~g}, 66 \%)$ as a colorless syrup, $R_{\mathrm{f}}$ (petroleum ether/EtOAc $\left.4: 1\right)=$ 0.20. The glucal $(890 \mathrm{mg}, 3.3 \mathrm{mmol})$ was dissolved in $\mathrm{CH}_{2} \mathrm{Cl}_{2}(15 \mathrm{~mL})$ and $\mathrm{AcOH}(0.35 \mathrm{~mL})$ and $\mathrm{PPh}_{3} \bullet \mathrm{HBr}(150 \mathrm{mg}, 0.44 \mathrm{mmol})$ was added. After stirring for $16 \mathrm{~h}$ at room temperature, more $\mathrm{PPh}_{3} \bullet \mathrm{HBr}(150 \mathrm{mg}, 0.44 \mathrm{mmol})$ was added, and stirring was continued for $24 \mathrm{~h}$. The solution was evaporated and purified by flash chromatography (petroleum ether/EtOAc 3:1) to afford 1,3,4,6-tetra$O$-acetyl-L-glucose $(643 \mathrm{mg}, 59 \%)$ as a colorless syrup (mixture of anomers; $R_{\mathrm{f}}$ (petroleum ether/EtOAc 2:1 $)=0.60)$. The tetracetate $(640 \mathrm{mg}, 1.9 \mathrm{mmol})$ was dissolved in $\mathrm{CH}_{2} \mathrm{Cl}_{2}(15 \mathrm{~mL}), \mathrm{PhSH}$ $(0.23 \mathrm{~mL}, 2.3 \mathrm{mmol})$ and $\mathrm{BF}_{3} \bullet \mathrm{OEt}_{2}(0.30 \mathrm{~mL}, 2.4 \mathrm{mmol})$ was added, and the mixture was stirred at 
room temperature for $1 \mathrm{~h}$. The solution was diluted with $\mathrm{CH}_{2} \mathrm{Cl}_{2}(150 \mathrm{~mL})$, washed with satd. aq. $\mathrm{NaHCO}_{3}$ solution, aq. $\mathrm{NaOH}$ solution $(10 \%)$, and brine. The organic phase was dried $\left(\mathrm{MgSO}_{4}\right)$, filtered, evaporated, and purified by flash chromatography (petroleum ether/EtOAc 6:1 $\rightarrow$ 4:1) to give thioglycoside S11 (590 mg, 80\%) as a colorless syrup (mixture of anomers, $R_{\mathrm{f}}$ (petroleum ether/EtOAc $4: 1)=0.43) ;$ LRMS (ESI) for $\mathrm{C}_{18} \mathrm{H}_{22} \mathrm{O}_{7} \mathrm{~S}(382.43): 383[\mathrm{M}+\mathrm{H}]^{+}$.

${ }^{1} \mathrm{H}$ NMR (400 MHz, $\left.\mathrm{CDCl}_{3}\right): \delta 7.45-7.20(\mathrm{~m}, 5 \mathrm{H}, \mathrm{Ph}), 5.64\left(\mathrm{~d}, J_{1,2 \mathrm{~b}}=5.71 \mathrm{~Hz}, 1 \alpha-\mathrm{H}\right), 5.23\left(\mathrm{ddd}, J_{2 \mathrm{a}, 3}=\right.$ $\left.5.2, J_{2 \mathrm{~b}, 3}=11.7, J_{3,4}=9.4 \mathrm{~Hz}, 3 \alpha-\mathrm{H}\right), 5.00-4.90(\mathrm{~m}, 3 \beta-\mathrm{H}, 4 \alpha-\mathrm{H}, 4 \beta-\mathrm{H}), 4.78\left(\mathrm{dd}, J_{1,2}=2.2, J_{1,2}=11.9\right.$ $\mathrm{Hz}, 1 \beta-\mathrm{H}), 4.48\left(\mathrm{ddd}, J_{4,5}=10.1, J_{5,6 \mathrm{a}}=5.3, J_{5,6 \mathrm{~b}}=2.1 \mathrm{~Hz}, 5 \alpha-\mathrm{H}\right), 4.28\left(\mathrm{dd}, J_{5,6 \mathrm{a}}=5.3, J_{6,6}=12.3 \mathrm{~Hz}\right.$, $\left.6 \alpha-\mathrm{H}_{\mathrm{a}}\right), 4.21\left(\mathrm{dd}, J_{5,6 \mathrm{a}}=5.5, J_{6,6}=12.2 \mathrm{~Hz}, 6 \beta-\mathrm{H}_{\mathrm{a}}\right), 4.10\left(\mathrm{dd}, J_{5,6 \mathrm{~b}}=2.3, J_{6,6}=12.2 \mathrm{~Hz}, 6 \beta-\mathrm{H}_{\mathrm{b}}\right), 3.98(\mathrm{dd}$, $\left.J_{5,6 \mathrm{~b}}=2.1, J_{6,6}=12.3 \mathrm{~Hz}, 6 \alpha-\mathrm{H}_{\mathrm{b}}\right), 3.61\left(\mathrm{ddd}, J_{4,5}=9.8, J_{5,6 \mathrm{a}}=5.5, J_{5,6 \mathrm{~b}}=2.3 \mathrm{~Hz}, 5 \beta-\mathrm{H}\right), 2.44-2.38(\mathrm{~m}$, $\left.2 \alpha-\mathrm{H}_{\mathrm{a}}, 2 \beta-\mathrm{H}_{\mathrm{a}}\right), 2.15\left(\mathrm{ddd}, J_{1,2 \mathrm{~b}}=5.9, J_{2 \mathrm{a}, 2 \mathrm{~b}}=13.5, J_{2 \mathrm{~b}, 3}=11.7 \mathrm{~Hz}, 2 \alpha-\mathrm{H}_{\mathrm{b}}\right), 2.03,2.02,1.99,1.98(6 \mathrm{~s}, \mathrm{Ac})$, $1.84-1.76\left(\mathrm{~m}, 2 \beta-\mathrm{H}_{\mathrm{b}}\right)$.

Thymidine 5'-(3,4,6-tri-O-acetyl-2-deoxy- $\beta$-L-glucopyranosyl diphosphate) (S12): TDP sugar S12 was prepared from thioglycoside $\mathbf{S 1 1}(46 \mathrm{mg}, 120 \mu \mathrm{mol})$ as described for $\mathbf{S 5}$. The resulting mixture of anomers $(\mathbf{S 1 2} \boldsymbol{\beta} / \mathbf{S 1 2} \boldsymbol{\alpha}=2: 1)$, was purified by preparative HPLC. Evaporation of the fractions containing the $\beta$-isomer gave $\mathbf{S 1 2}(18 \mathrm{mg}, 22 \%)$ as a powder upon lyophilization; $R_{\mathrm{t}}(\mathrm{TDP})=3.6 \mathrm{~min}$, $R_{\mathrm{t}}(\mathbf{S 1 2}, \beta$-anomer $)=9.5 \min , R_{\mathrm{t}}(\mathbf{S 1 2}, \alpha$-anomer $)=9.2 \min$, method A; LRMS $(\mathrm{ESI})$ for $\mathrm{C}_{22} \mathrm{H}_{32} \mathrm{~N}_{2} \mathrm{O}_{18} \mathrm{P}_{2}$ (674.44): $673[\mathrm{M}-\mathrm{H}]^{-}$.

${ }^{1} \mathrm{H}$ NMR (400 MHz, CD $\left.\mathrm{OD}\right): \delta 7.83(\mathrm{~s}, 1 \mathrm{H}, 6-\mathrm{H}), 6.32\left(\mathrm{t}, 1 \mathrm{H}, J_{1,2 \mathrm{a}}=J_{1,2 \mathrm{~b}}=6.4 \mathrm{~Hz}, 11^{\prime}-\mathrm{H}\right) 5.42(\mathrm{t}, 1 \mathrm{H}, J-$ $\left.{ }_{1,2 \mathrm{~b}}=J_{1, \mathrm{P}}=8.8 \mathrm{~Hz}, 1^{\prime \prime}-\mathrm{H}\right), 5.22-5.18\left(\mathrm{~m}, 1 \mathrm{H}, 3^{\prime \prime}-\mathrm{H}\right), 4.90\left(\mathrm{t}, 1 \mathrm{H}, J_{3,4}=J_{4,5}=9.6 \mathrm{~Hz}, 4 "-\mathrm{H}\right), 4.56-4.50(\mathrm{~m}$, $\left.1 \mathrm{H}, 3^{\prime}-\mathrm{H}\right), 4.35-4.10\left(\mathrm{~m}, 4 \mathrm{H}, 5^{\prime}-\mathrm{H}_{2}, 6^{\prime \prime}-\mathrm{H}_{2}\right), 4.05-4.01$ (m, 1H, 4'-H), 3.82-3.78 (m, 1H, 5"-H), 2.58-2.40 $\left(\mathrm{m}, 1 \mathrm{H}, 2^{\prime \prime}-\mathrm{H}_{\mathrm{a}}\right), 2.32-2.18\left(\mathrm{~m}, 2 \mathrm{H}, 2^{\prime}-\mathrm{H}_{2}\right), 2.04,2.00,1.94,1.92$ (4 s, each 3H, 3 Ac, thymidine $\left.\mathrm{CH}_{3}\right)$, 1.74-1.68 (m, 1H, 2"- $\left.\mathrm{H}_{\mathrm{b}}\right) ;{ }^{31} \mathrm{P}$ NMR (162 MHz, $\left.\mathrm{CD}_{3} \mathrm{OD}\right): \delta-10.2,-12.7$. 
Thymidine 5'-(2-deoxy- $\beta$-L-glucopyranosyl diphosphate) (11): The protected TDP sugar S12 (15 mg, $22 \mu \mathrm{mol})$ was dissolved in $\mathrm{MeOH} / \mathrm{H}_{2} \mathrm{O} / \mathrm{Et}_{3} \mathrm{~N}(1 \mathrm{~mL}, 2: 2: 1)$, stirred for $3 \mathrm{~h}$ at room temperature, evaporated, and purified by preparative HPLC to afford $11(8 \mathrm{mg}, 66 \%)$ as a white powder upon lyophilization; $R_{\mathrm{t}}=6.2$ min, method A; LRMS (ESI) for $\mathrm{C}_{16} \mathrm{H}_{26} \mathrm{~N}_{2} \mathrm{O}_{14} \mathrm{P}_{2}(532.33): 531[\mathrm{M}-\mathrm{H}]^{-}$.

${ }^{1} \mathrm{H}$ NMR $\left(400 \mathrm{MHz}, \mathrm{D}_{2} \mathrm{O}\right): \delta 7.75(\mathrm{~s}, 1 \mathrm{H}, 6-\mathrm{H}), 6.34\left(\mathrm{t}, 1 \mathrm{H}, J_{1,2 \mathrm{a}}=J_{1,2 \mathrm{~b}}=6.4 \mathrm{~Hz}, 1\right.$ '-H), $5.28(\mathrm{ddd}, 1 \mathrm{H}, J-$ $\left.{ }_{1,2 \mathrm{a}}=2.2, J_{1,2 \mathrm{~b}}=9.9, J_{1, \mathrm{P}}=8.0 \mathrm{~Hz}, 1^{\prime \prime}-\mathrm{H}\right), 4.60-4.56\left(\mathrm{~m}, 3^{\prime}-\mathrm{H}\right), 4.16-4.12\left(\mathrm{~m}, 3 \mathrm{H}, 4^{\prime}-\mathrm{H}_{,} 5^{\prime}-\mathrm{H}_{2}\right), 3.90(\mathrm{dd}$, $\left.1 \mathrm{H}, J_{5,6}=2.2, J_{6,6}=12.4 \mathrm{~Hz}, 6 "-\mathrm{H}_{\mathrm{a}}\right), 3.76-3.66\left(\mathrm{~m}, 2 \mathrm{H}, 3 "-\mathrm{H}, 6 "-\mathrm{H}_{\mathrm{b}}\right), 3.42(\mathrm{~m}, 1 \mathrm{H}, 5 "-\mathrm{H}), 4.24(\mathrm{t}, 1 \mathrm{H}$, $\left.J_{3,4}=J_{4,5}=9.6 \mathrm{~Hz}, 4 "-\mathrm{H}\right), 2.40-2.24\left(\mathrm{~m}, 3 \mathrm{H}, 2^{\prime}-\mathrm{H}_{2}, 2^{\prime \prime}-\mathrm{H}_{\mathrm{a}}\right), 1.91\left(\mathrm{~s}, 3 \mathrm{H}\right.$, thymidine $\left.\mathrm{CH}_{3}\right), 1.61(\mathrm{dt}, 1 \mathrm{H}$, $\left.J_{1,2}=9.9, J_{2,2}=J_{2,3}=12.1 \mathrm{~Hz}, 2^{\prime \prime}-\mathrm{H}_{\mathrm{b}}\right), 1.11 ;{ }^{31} \mathrm{P} \mathrm{NMR}\left(162 \mathrm{MHz}, \mathrm{D}_{2} \mathrm{O}\right): \delta-10.6,-12.7$.

TDP L-ristosamine (Thymidine 5'-(3-amino-2,3,6-deoxy-L-ribo-hexopyranosyl diphosphate), 12):
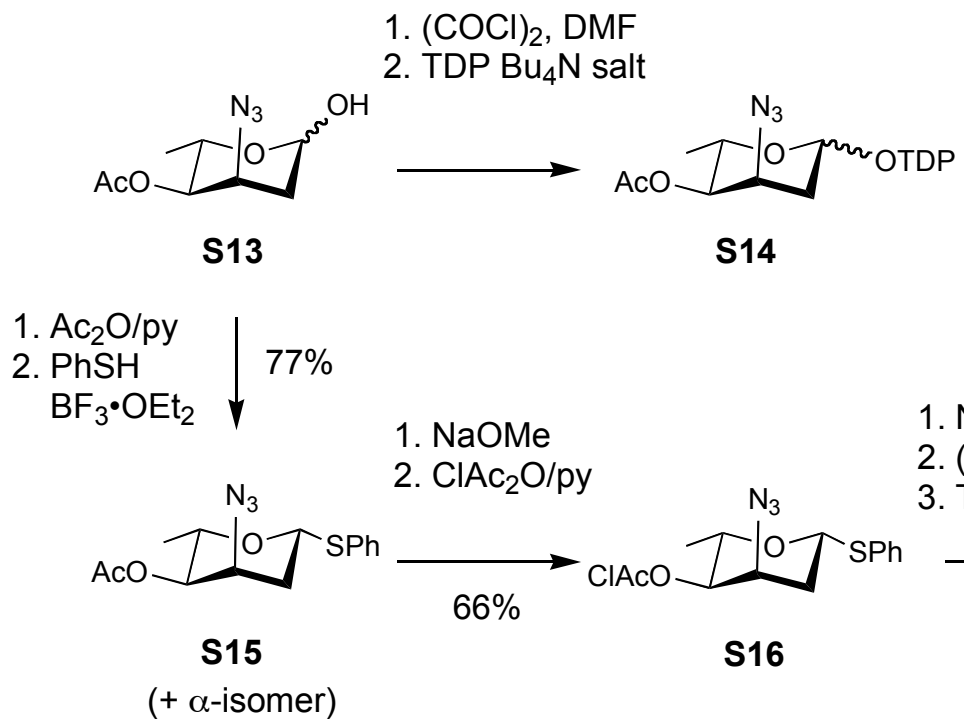

1. NIS

2. $(\mathrm{COCl})_{2}$, DMF

3. TDP $\mathrm{Bu}_{4} \mathrm{~N}$ salt

or $\mathrm{NaOMe}$

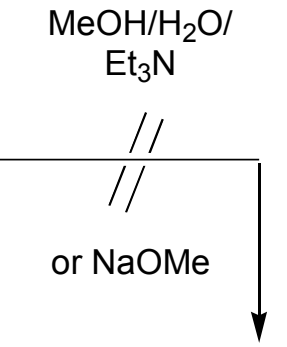

For the synthesis of TDP sugar 12, initially the known lactol $\mathbf{S 1 3}{ }^{\mathrm{S} 4}$ was converted to TDP sugar S14, which proved to be unstable to the basic reaction conditions required to deprotect the acetyl group. 
Therefore, O-3 was protected with the more base-labile chloroacetyl group, which was stable under the TDP coupling conditions and during HPLC purification using solvents containing $0.1 \% \mathrm{NH}_{4} \mathrm{HCO}_{3}$, but was cleaved upon standing and evaporation of the product-containing fractions, thus affording the 4deprotected S17 directly.

Phenyl 4-O-acetyl-3-azido-2,3,6-deoxy-1-thio-L-ribo-hexopyranoside (S15): Lactol S13 ${ }^{\mathrm{S} 4}$ (2.1 g, 10 mmol) was dissolved in pyridine $(30 \mathrm{~mL})$ and acetic anhydride $(15 \mathrm{~mL})$ and stirred at room temperature for $16 \mathrm{~h}$. The reaction mixture was evaporated, redissolved in $\mathrm{CH}_{2} \mathrm{Cl}_{2}(300 \mathrm{~mL})$ and washed with with $1 \mathrm{~N} \mathrm{HCl}$, satd. aq. $\mathrm{NaHCO}_{3}$ solution, and brine. The organic phase was dried $\left(\mathrm{MgSO}_{4}\right)$, filtered, evaporated, and purified by flash chromatography (petroleum ether/EtOAc 6:1) to give the diacetate $(2.44 \mathrm{~g}, 95 \%)$ as a colorless syrup, $R_{\mathrm{f}}=0.40$ (petroleum ether/EtOAc 6:1). The diacetate $(0.84 \mathrm{~g}, 3.3 \mathrm{mmol})$ was dissolved in $\mathrm{CH}_{2} \mathrm{Cl}_{2}(20 \mathrm{~mL})$, $\mathrm{PhSH}(0.39 \mathrm{~mL}, 3.9 \mathrm{mmol})$ and $\mathrm{BF}_{3} \bullet \mathrm{OEt}_{2}(0.51 \mathrm{~mL}, 4.1 \mathrm{mmol})$ was added, and the mixture was stirred at room temperature for $1 \mathrm{~h}$. The solution was diluted with $\mathrm{CH}_{2} \mathrm{Cl}_{2}(200 \mathrm{~mL})$, washed with satd. aq. $\mathrm{NaHCO}_{3}$ solution, aq. $\mathrm{NaOH}$ solution (10\%), and brine. The organic phase was dried $\left(\mathrm{MgSO}_{4}\right)$, filtered, evaporated, and purified by flash chromatography (petroleum ether/EtOAc 9:1 $\rightarrow$ 6:1). The two anomers of thioglycoside S15 could be separated; the $\beta$-isomer of $\mathbf{S 1 5}(610 \mathrm{mg})$ eluted first and was the major product, $R_{\mathrm{f}}=0.55$ (petroleum ether/EtOAc 6:1), the $\alpha$-anomer of $\mathbf{S 1 5}\left(190 \mathrm{mg}\right.$ ) eluted second, $R_{\mathrm{f}}=0.33$ (petroleum ether/EtOAc 6:1), combined yield: 81\%; LRMS (ESI) for $\mathrm{C}_{14} \mathrm{H}_{17} \mathrm{~N}_{3} \mathrm{O}_{3} \mathrm{~S}$ (307.37): 308 [M+H] .

S15 $\beta:{ }^{1} \mathrm{H}$ NMR $\left(400 \mathrm{MHz}, \mathrm{CDCl}_{3}\right): \delta$ 7.45-7.20 (m, 5H, Ph), $4.99\left(\mathrm{dd}, 1 \mathrm{H}, J_{1,2 \mathrm{a}}=2.1, J_{1,2 \mathrm{~b}}=11.5 \mathrm{~Hz}, 1-\right.$ $\mathrm{H}), 4.61\left(\mathrm{dd}, 1 \mathrm{H}, J_{3,4}=3.3, J_{4,5}=9.8 \mathrm{~Hz}, 4-\mathrm{H}\right), 4.16\left(\mathrm{q}, 1 \mathrm{H}, J_{2 \mathrm{a}, 3}=J_{2 \mathrm{~b}, 3}=J_{3,4}=3.3 \mathrm{~Hz}, 3-\mathrm{H}\right), 3.95(\mathrm{dq}$, $\left.1 \mathrm{H}, J_{4,5}=9.8, J_{5,6}=6.2 \mathrm{~Hz}, 5-\mathrm{H}\right), 2.10\left(\mathrm{ddd}, 1 \mathrm{H}, J_{1,2 \mathrm{a}}=2.2, J_{2 \mathrm{a}, 2 \mathrm{~b}}=14.2, J_{2 \mathrm{a}, 3}=3.3 \mathrm{~Hz}, 2-\mathrm{H}_{\mathrm{a}}\right), 2.08(\mathrm{~s}$, $3 \mathrm{H}, \mathrm{Ac}), 1.96\left(\mathrm{ddd}, 1 \mathrm{H}, J_{1,2 \mathrm{~b}}=11.5, J_{2 \mathrm{a}, 2 \mathrm{~b}}=14.2, J_{2 \mathrm{~b}, 3}=3.3 \mathrm{~Hz}, 2-\mathrm{H}_{\mathrm{b}}\right), 1.21\left(\mathrm{~d}, J_{5,6}=6.2 \mathrm{~Hz}, 6-\mathrm{H}_{3}\right)$. 
Phenyl 4-O-chloroacetyl-3-azido-2,3,6-deoxy-1-thio- $\beta$-L-ribo-hexopyranoside (S16): The $\beta$-isomer of thioglycoside S15 (160 mg, $0.52 \mathrm{mmol})$ was dissolved in $\mathrm{MeOH}$ (10 mL) containing NaOMe (0.22 $\mathrm{mL}, 0.11 \mathrm{mmol}, 0.5 \mathrm{M}$ in $\mathrm{MeOH}$ ) and stirred for $3 \mathrm{~h}$. The solution was neutralized with Amberlite IR $120\left(\mathrm{H}^{+}\right.$form $)$, filtered, evaporated and coevaporated with toluene $(3 \mathrm{x})$. The residue $\left(R_{\mathrm{f}}\right.$ (petroleum ether/EtOAc $4: 1)=0.22)$, was dissolved in pyridine $(3 \mathrm{~mL})$, cooled to $0^{\circ} \mathrm{C}$, and treated with chloroacetic acid anhydride $(170 \mathrm{mg}, 1 \mathrm{mmol})$. After stirring for $3 \mathrm{~h}$ at this temperature, $\mathrm{MeOH}(2 \mathrm{~mL})$ was added, and the reaction mixture was evaporated. The residue was redissolved in $\mathrm{CH}_{2} \mathrm{Cl}_{2}(100 \mathrm{~mL})$ and washed with with $1 \mathrm{~N} \mathrm{HCl}$, satd. aq. $\mathrm{NaHCO}_{3}$ solution, and brine. The organic phase was dried $\left(\mathrm{MgSO}_{4}\right)$, filtered, evaporated, and purified by flash chromatography (petroleum ether/EtOAc 10:1) to give chloroacetate $\mathbf{S 1 6}(117 \mathrm{mg}, 66 \%)$ as a colorless syrup; $R_{\mathrm{f}}$ (petroleum ether/EtOAc 9:1) = 0.50; LRMS (ESI) for $\mathrm{C}_{14} \mathrm{H}_{16} \mathrm{ClN}_{3} \mathrm{O}_{3} \mathrm{~S}(341.81): 342[\mathrm{M}+\mathrm{H}]^{+}$.

${ }^{1} \mathrm{H}$ NMR (400 MHz, $\left.\mathrm{CDCl}_{3}\right): \delta 7.47-7.22(\mathrm{~m}, 5 \mathrm{H}, \mathrm{Ph}), 5.00\left(\mathrm{dd}, 1 \mathrm{H}, J_{1,2 \mathrm{a}}=2.1, J_{1,2 \mathrm{~b}}=11.5 \mathrm{~Hz}, 1-\mathrm{H}\right)$, $4.68\left(\mathrm{dd}, 1 \mathrm{H}, J_{3,4}=3.3, J_{4,5}=9.8 \mathrm{~Hz}, 4-\mathrm{H}\right), 4.18\left(\mathrm{q}, 1 \mathrm{H}, J_{2 \mathrm{a}, 3}=J_{2 \mathrm{~b}, 3}=J_{3,4}=3.3 \mathrm{~Hz}, 3-\mathrm{H}\right), 4.02(\mathrm{~d}, 2 \mathrm{H}$, $\mathrm{ClAc}), 3.98\left(\mathrm{dq}, 1 \mathrm{H}, J_{4,5}=9.8, J_{5,6}=6.2 \mathrm{~Hz}, 5-\mathrm{H}\right), 2.15\left(\mathrm{ddd}, 1 \mathrm{H}, J_{1,2 \mathrm{a}}=2.2, J_{2 \mathrm{a}, 2 \mathrm{~b}}=14.2, J_{2 \mathrm{a}, 3}=3.3 \mathrm{~Hz}\right.$, $\left.2-\mathrm{H}_{\mathrm{a}}\right), 1.99\left(\mathrm{ddd}, 1 \mathrm{H}, J_{1,2 \mathrm{~b}}=11.5, J_{2 \mathrm{a}, 2 \mathrm{~b}}=14.2, J_{2 \mathrm{~b}, 3}=3.3 \mathrm{~Hz}, 2-\mathrm{H}_{\mathrm{b}}\right), 1.22\left(\mathrm{~d}, J_{5,6}=6.2 \mathrm{~Hz}, 6-\mathrm{H}_{3}\right)$.

TDP 3-azido L-ristosamine (Thymidine 5'-(3-azido-2,3,6-deoxy-L-ribo-hexopyranosyl diphosphate), S17): TDP sugar S17 was prepared from thioglycoside $\mathbf{S 1 6}(56 \mathrm{mg}, 160 \mu \mathrm{mol}$ ) as described for S5. The TDP coupling reaction was followed by analytical HPLC and showed the formation of the 4chloroacetyl derivative of $\mathbf{S 1 7}, R_{\mathrm{t}}=10.9,10.3 \min (\alpha$ - and $\beta$-anomer $), R_{\mathrm{t}}(\mathrm{TDP})=3.6 \min$, method A. The chloroacetyl group was cleaved in part while standing and completely upon evaporation of the product containing fractions to give the TDP sugar S17 (30 mg, mixture of anomers $(\beta / \alpha=4: 3), 33 \%)$ as a white powder upon lyophilization; LRMS (ESI) for $\mathrm{C}_{16} \mathrm{H}_{25} \mathrm{~N}_{5} \mathrm{O}_{13} \mathrm{P}_{2}$ (557.34): $556[\mathrm{M}-\mathrm{H}]^{-}$. S17ß: ${ }^{1} \mathrm{H}$ NMR (500 MHz, CD $\left.30 D\right): \delta 7.63(\mathrm{~s}, 1 \mathrm{H}, 6-\mathrm{H}), 6.20\left(\mathrm{t}, 1 \mathrm{H}, J_{1,2 \mathrm{a}}=J_{1,2 \mathrm{~b}}=6.2 \mathrm{~Hz}, 1^{\prime}-\mathrm{H}\right), 5.21$ $\left(\mathrm{dt}, 1 \mathrm{H}, J_{1,2 \mathrm{a}}=2.0, J_{1,2 \mathrm{~b}}=J_{1, \mathrm{P}}=9.5 \mathrm{~Hz}, 1^{\prime \prime}-\mathrm{H}\right), 4.64-4.60\left(\mathrm{~m}, 1 \mathrm{H}, 3^{\prime}-\mathrm{H}\right), 4.06-3.92\left(\mathrm{~m}, 4 \mathrm{H}, 4^{\prime}-\mathrm{H}, 5^{\prime}-\mathrm{H}_{2}\right.$, 
3"-H), 3.39 (dq, $\left.1 \mathrm{H}, J_{4,5}=9.8, J_{5,6}=6.6 \mathrm{~Hz}, 5 "-\mathrm{H}\right), 3.40\left(\mathrm{dd}, 1 \mathrm{H}, J_{3,4}=3.6, J_{4,5}=9.8 \mathrm{~Hz}, 4 "-\mathrm{H}\right), 2.25-$ $2.18\left(\mathrm{~m}, 2 \mathrm{H}, 2^{\prime}-\mathrm{H}_{2}\right), 2.10\left(\mathrm{ddd}, 1 \mathrm{H}, J_{1,2 \mathrm{a}}=2.0, J_{2 \mathrm{a}, 2 \mathrm{~b}}=14.2, J_{2 \mathrm{a}, 3}=3.2 \mathrm{~Hz}, 2^{\prime \prime}-\mathrm{H}_{\mathrm{a}}\right), 1.79(\mathrm{~s}, 3 \mathrm{H}$, thymidine $\left.\mathrm{CH}_{3}\right), 1.70\left(\mathrm{ddd}, 1 \mathrm{H}, J_{1,2 \mathrm{~b}}=9.5, J_{2 \mathrm{a}, 2 \mathrm{~b}}=14.2, J_{2 \mathrm{~b}, 3}=3.2 \mathrm{~Hz}, 2 "-\mathrm{H}_{\mathrm{b}}\right), 1.10\left(\mathrm{~d}, 3 \mathrm{H}, J_{5,6}=6.6 \mathrm{~Hz}, 6 "-\mathrm{H}_{3}\right) ;{ }^{31} \mathrm{P}$ NMR (162 MHz, $\left.\mathrm{CD}_{3} \mathrm{OD}\right): \delta-10.6,-12.9$.

TDP L-ristosamine (Thymidine 5'-(3-amino-2,3,6-deoxy-L-ribo-hexopyranosyl diphosphate), 12): The azido TDP sugar $\mathbf{S 1 7}(10 \mathrm{mg}, 18 \mu \mathrm{mol})$ was dissolved in $\mathrm{MeOH} / \mathrm{H}_{2} \mathrm{O} / \mathrm{Et}_{3} \mathrm{~N}(1 \mathrm{~mL}, 2: 2: 1)$, TCEP (26 $\mathrm{mg}, 90 \mu \mathrm{mol})$ was added, and the reaction was stirred for $2 \mathrm{~h}$ at room temperature. Evaporation and purification by preparative HPLC afforded $12(7 \mathrm{mg}$, mixture of anomers $(\beta / \alpha=4: 3), 70 \%)$ as a white powder upon lyophilization; $R_{\mathrm{t}}=6.3,6.7$ min, method A; LRMS (ESI) for $\mathrm{C}_{16} \mathrm{H}_{27} \mathrm{~N}_{3} \mathrm{O}_{13} \mathrm{P}_{2}$ (531.35): 530 $[\mathrm{M}-\mathrm{H}]^{-}$.

12ß: ${ }^{1} \mathrm{H}$ NMR (500 MHz, $\left.\mathrm{D}_{2} \mathrm{O}\right): \delta 7.58(\mathrm{~s}, 1 \mathrm{H}, 6-\mathrm{H}), 6.20\left(\mathrm{t}, 1 \mathrm{H}, J_{1,2 \mathrm{a}}=J_{1,2 \mathrm{~b}}=6.2 \mathrm{~Hz}, 1^{\prime}-\mathrm{H}\right), 5.54(\mathrm{~m}$, 1H, $1 "-\mathrm{H}), 4.68-4.60\left(\mathrm{~m}, 1 \mathrm{H}, 3\right.$ '-H), 4.05-3.95 (m, 3H, 4'-H, 5'- $\left.\mathrm{H}_{2}\right), 3.80-3.40$ (m, 2H, 4"-H, 5"-H), 2.25-2.10 (m, 3H, 2'- $\left.\mathrm{H}_{2}, 2 "-\mathrm{H}_{\mathrm{a}}\right), 1.78\left(\mathrm{~s}, 3 \mathrm{H}\right.$, thymidine $\left.\mathrm{CH}_{3}\right), 1.78-1.74\left(\mathrm{~m}, 2^{\prime \prime}-\mathrm{H}_{\mathrm{b}}\right), 1.12\left(\mathrm{~d}, 3 \mathrm{H}, J_{5,6}=\right.$ $\left.6.6 \mathrm{~Hz}, 6 "-\mathrm{H}_{3}\right) ;{ }^{31} \mathrm{P}$ NMR $\left(162 \mathrm{MHz}, \mathrm{D}_{2} \mathrm{O}\right): \delta-10.7,-12.8$.

(S1) Oberthür, M.; Leimkuhler, C.; Kahne, D. Org. Lett. 2004, 6, 2873-2876.

(S2) Mioskowski, C.; Bolitt, V.; Lee, S.-G.; Falck, J. R.; J. Org. Chem. 1990, 55, 5812-5813.

(S3) Gildersleeve, J.; Smith, A.; Sakurai, K.; Raghavan, S.; Kahne, D. J. Am. Chem. Soc. 1999, 121, 6176-6182.

(S4) Abbaci, B.; Florent, J.-C.; Monneret, C. Bull. Soc. Chim. France 1989, 669-672. 